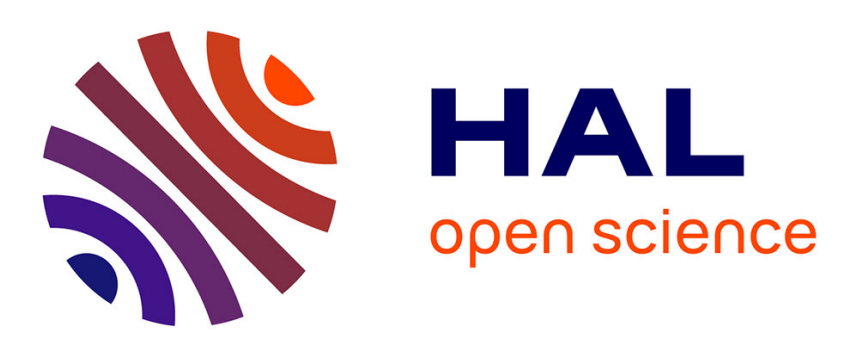

\title{
Transformation Thermomechanics of Alloy Materials in the Process of Martensitic Transformation: A Unified Theory
}

\author{
K. Tanaka, F. Nishimura, F. Fischer, E. Oberaigner
}

\section{- To cite this version:}

K. Tanaka, F. Nishimura, F. Fischer, E. Oberaigner. Transformation Thermomechanics of Alloy Materials in the Process of Martensitic Transformation: A Unified Theory. Journal de Physique IV Proceedings, 1996, 06 (C1), pp.C1-455-C1-463. 10.1051/jp4:1996144 • jpa-00254176

\section{HAL Id: jpa-00254176 https://hal.science/jpa-00254176}

Submitted on 1 Jan 1996

HAL is a multi-disciplinary open access archive for the deposit and dissemination of scientific research documents, whether they are published or not. The documents may come from teaching and research institutions in France or abroad, or from public or private research centers.
L'archive ouverte pluridisciplinaire HAL, est destinée au dépôt et à la diffusion de documents scientifiques de niveau recherche, publiés ou non, émanant des établissements d'enseignement et de recherche français ou étrangers, des laboratoires publics ou privés. 


\title{
Transformation Thermomechanics of Alloy Materials in the Process of Martensitic Transformation: A Unified Theory
}

\author{
K. Tanaka, F. Nishimura, F.D. Fischer* and E.R. Oberaigner** \\ Department of Aerospace Engineering, Tokyo Metropolitan Institute of Technology, Asahigaoka 6-6, 191 \\ Hino, Tokyo, Japan \\ * Institute of Mechanics and Christian Doppler Laboratory for Micromechanics of Materials, University for \\ Mining and Metallurgy, Franz-Jozef Strasse 18, 8700 Leoben, Austria \\ ** Institute of Mechanics, University for Mining and Metallurgy, Franz-Jozef Strasse 18, 8700 Leoben, \\ Austria
}

\begin{abstract}
A unified theory on the transformation and thermomechanical behavior of alloy materials is proposed in the processes of plastic deformation and martensitic transformation. A transformation condition is introduced to describe the start and progress of the transformation, together with the yield condition for plasticity. The constitutive equations, thermomechanical and calorimetric, are derived following the conventional continuum mechanics whereas the evolutional equations for the internal variables are oblained by solving a conditional extremum problem. The heat conduction equation is presented. The transformation kinetics is discussed to reach Magee's kinetics.
\end{abstract}

\section{INTRODUCTION}

Transformation Thermomechanics is devoted to describing the thermomechanical behavior of the materials in the process of transformations. Its final task might be to provide the effective methodology, microscopic and macroscopic, of designing the materials with the help of the transformation phenomena to meet the required specifications, on the strength and toughness, for example. It is first requested to establish a theoretical framework which describes well the material response during transformations. When carrying out a successful heat treatment, the engineers not only need the macroscopic quantities induced in the materials such as the stress, strain, temperature and the volume fraction of the phases but also ask the information about the microscopic structural changes due to the metallurgical processes undergoing in the materials. The materials may deform plastically in the processes considered as is the usual cases in the application of the TRIP (transformation-induced plasticity) steels. The factors to be considered are so many when thinking about the enginecring application of the theory.

The martensitic transformation is one of the topics to be investigated in transformation thermomechanics since it plays an important role in many engineering problems such as, for example, the material enhancement, shape memory effect and the transformation toughening. Intensive studies have been done in this field with their own material modeling. Müller and coworkers [1,2] have established a theory based on the mixture theory with a non-convex free energy to predict the full and sub stress-strain hystereses under loading. The idea was generalized by Raniecki et al. [3] to the multiaxial and nonisothermal cases. Levitas [4] explained the experimental observation on the subsequent transformation start stress after the incomplete martensitic transformation by taking account of a new threshold value of the driving force. The propagation of the phase interface was discussed through the energy momentum tensor which relates to the driving force [5-8]. Starting from the energy balance and the Clausius-Duhem incquality Tanaka et al. [9] derived the thermomechanical and calorimetric constitutive equations in the shape memory alloys, which was reformulated by Brandon and Rogers [10] and Brinson [11] in the different metallurgical processes. The derivation of the material performance on the macroscopic level from the microscopic material response is a theme to be solved both in micromechanics and transformation thermomechanics. Fischer and Tanaka [12] attacked the problem by introducing two material structural levels; microregion and mesodomain. It should be noted the work of Sun et al. $[13,14]$ who tried to establish a unified theory of transformation and thermomechanics in order to explain the transformation toughening in ceramics.

In this study, following Tanaka et al. $[9,15]$, a unified theory on the macroscopic transformation and thermomechanical behavior of alloy materials is presented in the process of martensitic transformation. The alloy is assumed to deform plastically during transformation. The transformation condition is introduced to 
describe the start and progress of the transformation together with the conventional yield condition which governs the plastic process. The evolution equations for the irreversible processes are determined by solving a conditional extremum problem. A full set of constitutive relations is, together with the heat conduction equation, presented. The transformation kinetics is discussed in the case of no plastic deformation.

\section{MODELING OF MICROSTRUCTURES IN ALLOY MATERIALS}

The alloy materials, which are in the process of martensitic transformation, are assumed to have two levels of microscopic structure; the microregion and the mesodomain [12]. The microregion is defined as the smallest structural element, which experiences the transformation and the associated spontaneous response when its thermomechanical state reaches a critical condition. The microregion represents the microscopic alloy behavior at a domain characterized by a habit plane which could be identified by the Eulerian angles between the local coordinate system attached to the microregion and the global coordinate system attached to the sample specimen.

A mesodomain is composed of enough large number of microregions, but is still small compared to the size of the sample specimen. The ensemble averaging over the mesodomain produces the uniform mesoscopic material properties. The mesodomain, therefore, represents the material behavior of a material point in the sense of continuum mechanics. In this study the attention is focused on constructing a unified theory of the thermomechanical and transformation behavior of the materials on the level of mesodomain by taking into account the material performance on the level of microregions.

The microregions can be always well classified into two classes: those which are not yet transformed and those which have already been transformed from the parent phase to the martensite phase thermally or athermally. If the transformation state of each microregion is measured by means of the micro-fraction $\tilde{\xi}=$ 0 for the untransformed state or $\tilde{\xi}=1$ for the transformed state, one could well define a so-called macrofraction $\xi$, by averaging $\tilde{\xi}$ over the mesodomain, to characterize the extent of martensitic transformation on the mesoscopic level. The macro-fraction $\xi$ can be regarded as an internal variable in continuum mechanics to specify the mesoscopic transformation state.

In each microregion a strain is induced spontaneously when the transformation takes place. The strain is, after being averaged over the mesodomain, observed as a mesoscopic stress-free transformation strain tensor $E^{*}$. It should be noted that $E^{*}$ starts increasing just after the transformation starts at some microregions in the mesodomain. The microregions may exhibit the plastic deformation before the transformation as the parent phase, or just at the moment of the transformation or after it as the martensitic phase. Averaging these strains over the mesodomain one can assume that the elastic and plastic deformation state can be identified by the elastic strain and the plastic strain, respectively, on the level of the mesodomain. In order to describe strictly the situation, let us assume that the Green strain tensor $E$ can be additively decomposed in the rate form,

$$
\dot{E}=\dot{E}^{e}+\dot{E}^{p}+\dot{E}^{*}
$$

where $\dot{E}^{e}$ and $\dot{E}^{p}$ stand for the elastic and plastic components, respectively.

Similar dccomposition of the rate of the entropy density $\eta[16]$ is also assumed here by

$$
\dot{\eta}=\dot{\eta}^{e}+\dot{\eta}^{p}+\dot{\eta}^{*}
$$

The thermomechanical process is then understood to be fully specified by a set of state variables

$$
\boldsymbol{j}=\left(\boldsymbol{E}^{e}, \eta^{e} ; \xi, \boldsymbol{E}^{*}, \eta^{*} ; \kappa, \boldsymbol{E}^{p}, \eta^{p}\right),
$$

where $\kappa$ specifies the work-hardening of the materials. The last six variables are the internal state variables.

\section{DESCRIPTION OF TRANSFORMATION/DEFORMATION PROCESSES}

Starting from the energy balance and the Clausius-Duhem inequality, following the standard thermodynamic discussion, one can reach the constitutive equations in the elastic process and the dissipation incquality; 


$$
\begin{aligned}
& E^{e}=-\rho_{0} \frac{\partial \Psi}{\partial \Sigma}, \quad \eta^{e}=-\frac{\partial \Psi}{\partial T}, \\
& \mathscr{D}=K_{1} \dot{\xi}+K_{2}: \dot{E}^{*}+K_{3} \dot{\eta}^{*}+K_{4} \dot{\kappa}+K_{5}: \dot{E}^{p}+K_{6} \dot{\eta}^{p}-\frac{1}{T} Q \cdot \operatorname{Grad} T \geq 0,
\end{aligned}
$$

where $T$ denotes the temperature, and the Gibbs free energy $\Psi$, the second Piola-Kirchhoff stress tensor $\Sigma$ and the material heat flux $Q$ are introduced. The thermodynamic forces are defined by

$$
\begin{array}{lll}
K_{1}=-\rho_{0} \frac{\partial \Psi}{\partial \xi}, & K_{2}=\Sigma-\rho_{0} \frac{\partial \Psi}{\partial E^{*}}, & K_{3}=\rho_{0}\left(T-\frac{\partial \Psi}{\partial \eta^{*}}\right), \\
K_{4}=-\rho_{0} \frac{\partial \Psi}{\partial \kappa}, & K_{5}=\Sigma-\rho_{0} \frac{\partial \Psi}{\partial E^{p}}, & K_{6}=\rho_{0}\left(T-\frac{\partial \Psi}{\partial \eta^{p}}\right) .
\end{array}
$$

The thermodynamic force $K_{1}$ is called the driving force of transformation $[17,18]$ in metallurgy and the affinity in chemistry. For the sake of simplicity a notation

$$
\mathscr{D}=K_{t} * \dot{\boldsymbol{\kappa}}_{t}+K_{p} * \dot{\boldsymbol{\kappa}}_{p}-\frac{1}{T} Q \cdot \operatorname{Grad} T \geq 0,
$$

is employed in Eq.(4) 3 by introducing the following quantities: The generalized thermodynamic forces relating to the transformation and the plastic deformation, respectively,

$$
K_{t}=\left(K_{1}, K_{2}, K_{3}\right), \quad K_{p}=\left(K_{4}, K_{5}, K_{6}\right),
$$

the corresponding generalized internal variables and their material derivative

$$
\kappa_{t}=\left(\xi, E^{*}, \eta^{*}\right), \kappa_{p}=\left(\kappa, E^{p}, \eta^{p}\right), \dot{\kappa}_{t}=\left(\dot{\xi}, \dot{E}^{*}, \dot{\eta}^{*}\right), \dot{\kappa}_{p}=\left(\dot{\kappa}, \dot{E}^{p}, \dot{\eta}^{p}\right) .
$$

A generalized state variable $\Omega=(\Sigma, T)$ is also introduced for later use. The symbol * denotes the appropriate inner product between the corresponding quantities. It is worth noting here that the Gibbs free cnergy can be simply written by

$$
\Psi=\Psi\left(\Omega ; \kappa_{i} ; \kappa_{p}\right),
$$

and, thereforc, the same is true for the generalized thermodynamic forces

$$
\boldsymbol{K}_{t}=\boldsymbol{K}_{t}\left(\boldsymbol{\Omega} ; \boldsymbol{\kappa}_{t} ; \boldsymbol{\kappa}_{p}\right), \quad \boldsymbol{K}_{p}=\boldsymbol{K}_{p}\left(\boldsymbol{\Omega} ; \boldsymbol{\kappa}_{t} ; \boldsymbol{\kappa}_{p}\right) .
$$

From Eq.(4) 1,2 one can derive the thermomechanical and calorimetric constitutive equations in rate form if the elastic process is reasonably assumed not to be influenced by the irreversible processes

$$
\begin{aligned}
\dot{\boldsymbol{E}}^{e}=\boldsymbol{D}: \dot{\boldsymbol{\Sigma}}+\boldsymbol{\theta} \dot{\boldsymbol{T}}, & \dot{\eta}^{e}=\frac{\boldsymbol{\theta}}{\rho_{0}}: \dot{\boldsymbol{\Sigma}}+c \dot{T}, \\
D=-\rho_{0} \frac{\partial^{2} \Psi}{\partial \Sigma \otimes \partial \Sigma}, & \boldsymbol{\theta}=-\rho_{0} \frac{\partial^{2} \Psi}{\partial \Sigma \partial T}, \quad c=-\rho_{0} \frac{\partial^{2} \Psi}{\partial T},
\end{aligned}
$$

where the material parameters $D, \boldsymbol{\theta}$ and $c$ stand for the elastic moduli tensor, the thermoclastic tensor and the specific heat, respectively.

\section{CONSTITUTIVE EQUATIONS IN IRREVERSIBLE PROCESSES}

The conventional plasticity [19] requires that the plastic process must be restricted by a loading condition which may generally be given in the present context as

$$
f=f\left(\boldsymbol{\Omega} ; \boldsymbol{K}_{t}, \boldsymbol{K}_{p} ; \boldsymbol{\kappa}_{t}, \boldsymbol{\kappa}_{p}\right)=0 \text { and } \dot{f}=0 .
$$

Similarly, the transformation is assumed here to undergo only under a transformation condition 


$$
g=g\left(\boldsymbol{\Omega} ; K_{t}, K_{p} ; \boldsymbol{\kappa}_{t}, \boldsymbol{\kappa}_{p}\right)=0 \text { and } \dot{g}=0 .
$$

For the moment it is cnough to point out the fact that the condition (13) is often written by means of the thermodynamic force $K_{l}$ only [17,18]. It is also worthwhile to note that the time $t$ is included in Eq.(13) as an implicit parameter through the state variables, which reflects the fact that the martensitic transformation is of diffusionless.

The requirement of the second law of thermodynamics can be read as follows: The actual thermomechanical process maximize the dissipation $\mathscr{D}$ in Eq.(6) under the thermomechanical restrictions (12) and (13). The problem can, therefore, be interpreted as a conditional extremum problem of

$$
\begin{aligned}
\bar{D} & =\mathscr{D}-\dot{\lambda} f-\dot{\mu} g \\
& =K_{t} * \dot{\boldsymbol{x}}_{t}+K_{p} * \dot{\boldsymbol{x}}_{p}-\frac{1}{T} Q \cdot \operatorname{Grad} T-\dot{\lambda} f-\dot{\mu} g
\end{aligned}
$$

with the Lagrange multipliers $\dot{\lambda}$ and $\dot{\mu}$ [20-22]. The usual mathematical procedure leads to the final formulas

$$
\dot{\boldsymbol{\kappa}}_{t}=\dot{\lambda} \frac{\partial f}{\partial \boldsymbol{K}_{t}}+\dot{\mu} \frac{\partial g}{\partial \boldsymbol{K}_{t}}, \quad \dot{\boldsymbol{\kappa}}_{p}=\dot{\lambda} \frac{\partial f}{\partial \boldsymbol{K}_{p}}+\dot{\mu} \frac{\partial g}{\partial \boldsymbol{K}_{p}},
$$

which represent the evolution equations, or better to say, the constitutive equations for the generalized internal variables $\kappa_{t}$ and $\kappa_{p}$. Equation(15) 1 is explicitly read as

$$
\dot{\xi}=\dot{\lambda} \frac{\partial f}{\partial K_{1}}+\dot{\mu} \frac{\partial g}{\partial K_{1}}, \quad \dot{E}^{*}=\dot{\lambda} \frac{\partial f}{\partial K_{2}}+\dot{\mu} \frac{\partial g}{\partial K_{2}}, \quad \dot{\eta}^{*}=\dot{\lambda} \frac{\partial f}{\partial K_{3}}+\dot{\mu} \frac{\partial g}{\partial K_{3}} .
$$

Equation(16) ${ }_{1}$ is called the transformation kinetics in metallurgy [23], which governs the progress of transformation. The component equations in Eq.(15) 2 are written as follows:

$$
\dot{\kappa}=\dot{\lambda} \frac{\partial f}{\partial K_{4}}+\dot{\mu} \frac{\partial g}{\partial K_{4}}, \quad \dot{E}^{p}=\dot{\lambda} \frac{\partial f}{\partial K_{5}}+\dot{\mu} \frac{\partial g}{\partial K_{5}}, \quad \dot{\eta}^{p}=\dot{\lambda} \frac{\partial f}{\partial K_{6}}+\dot{\mu} \frac{\partial g}{\partial K_{6}} .
$$

Equation $(17)_{1}$ governs the work-hardening of the material while Eq.(17) 2 is called the thermomechanical flow rule in plasticity and Eq.(17) 3 the calorimetric flow rule. The second terms on the right-hand side in Eq.(17) represent the contribution from the transformation.

The first term in the right-hand side of Eq.(16),$\dot{\lambda} \frac{\partial f}{\partial K_{I}}$, states that the plastic process can activate the transformation, resulting in an irreversible strain rate $\dot{\lambda} \frac{\partial f}{\partial K_{2}}$ in Eq.(16) $)_{2}$ and an irreversible entropy density rate $\dot{\lambda} \frac{\partial f}{\partial K_{3}}$ in Eq.(16) $)_{3}$. The effect stems solely from the assumption that the plastic condition $f$ includes the thermodynamic force $K_{t}$ as the variables. On the other hand, the second term in the right-hand side of Eq. $(17)_{1}, \dot{\mu} \frac{\partial g}{\partial K_{4}}$, expresses that the work-hardening process is influenced by the progress of transformation. As a result an irreversible strain rate $\dot{\mu} \frac{\partial g}{\partial K_{5}}$ and an irreversible entropy density rate $\dot{\mu} \frac{\partial g}{\partial K_{\sigma}}$ are observed.

In the conventional transformation-induced plasticity $[24,25]$ the strain rate due to transformation is derived by assuming a loading function, $f$ in the present case, depending on the fraction of martensite $\xi$. It should be firstly noted that Eq.(12) is more general than their loading functions since it depends also on the state variables $E^{*}$ and $\eta^{*}$. The total irreversible strain rate $\dot{E}^{i r}$ in their case is given by a sum of the usual plastic part and the part due to the transformation, in our context, by

$$
\dot{\boldsymbol{E}^{i r}}=\dot{\lambda} \frac{\partial f}{\partial \boldsymbol{K}_{5}}+\dot{\lambda} \frac{\partial f}{\partial \boldsymbol{K}_{2}}
$$


which, of course, vanishes when the plastic process stops, i.e. when $\dot{\lambda}=0$. The theories, therefore, exclude another irreversible strain rates $\dot{\mu} \frac{\partial g}{\partial K_{5}}$ in Eq.(17) and $\dot{\mu} \frac{\partial g}{\partial K_{2}}$ in Eq.(16), which are induced by the transformation, totally inrelevant to the progress of plastic process. The present theory claims that the irreversible strain rate should be expressed by

$$
\dot{E}^{i r}=\dot{\lambda} \frac{\partial f}{\partial K_{2}}+\dot{\lambda} \frac{\partial f}{\partial K_{5}}+\dot{\mu} \frac{\partial g}{\partial K_{2}}+\dot{\mu} \frac{\partial g}{\partial K_{5}} .
$$

Actually, for example, the shape memory alloys are almost all cases used within the elastic range with no macroscopic plastic deformation [26], in which the strain rate $\dot{\mu} \frac{\partial g}{\partial K_{2}}$ plays a major role.

The Lagrange multipliers $\dot{\lambda}$ and $\dot{\mu}$ introduced in Eq.(14) can be determincd from the consistency conditions Eqs.(12) 2 and (13)2,

$$
\begin{gathered}
\dot{f=} \frac{\partial f}{\partial \boldsymbol{\Omega}} * \dot{\boldsymbol{\Omega}}+\frac{\partial f}{\partial \boldsymbol{K}_{t}} *\left(\frac{\partial \boldsymbol{K}_{t}}{\partial \boldsymbol{\Omega}} * \dot{\boldsymbol{\Omega}}+\frac{\partial \boldsymbol{K}_{t}}{\partial \boldsymbol{\kappa}_{t}} * \dot{\boldsymbol{\kappa}}_{t}+\frac{\partial \boldsymbol{K}_{t}}{\partial \boldsymbol{\kappa}_{p}} * \dot{\boldsymbol{\kappa}}_{p}\right) \\
+\frac{\partial f}{\partial \boldsymbol{K}_{p}} *\left(\frac{\partial \boldsymbol{K}_{p}}{\partial \boldsymbol{\Omega}} * \dot{\boldsymbol{\Omega}}+\frac{\partial \boldsymbol{K}_{p}}{\partial \boldsymbol{\kappa}_{t}} * \dot{\boldsymbol{\kappa}}_{t}+\frac{\partial \boldsymbol{K}_{p}}{\partial \boldsymbol{\kappa}_{p}} * \dot{\boldsymbol{\kappa}}_{p}\right) \\
+\frac{\partial f}{\partial \boldsymbol{\kappa}_{t}} * \dot{\boldsymbol{\kappa}}_{t}+\frac{\partial f}{\partial \boldsymbol{\kappa}_{p}} * \dot{\boldsymbol{\kappa}}_{p}=0, \\
\dot{g}=\frac{\partial g}{\partial \boldsymbol{\Omega}} * \dot{\boldsymbol{\Omega}}+\frac{\partial g}{\partial \boldsymbol{K}_{t}} *\left(\frac{\partial \boldsymbol{K}_{t}}{\partial \boldsymbol{\Omega}} * \dot{\boldsymbol{\Omega}}+\frac{\partial \boldsymbol{K}_{t}}{\partial \boldsymbol{\kappa}_{t}} * \dot{\boldsymbol{\kappa}}_{t}+\frac{\partial \boldsymbol{K}_{t}}{\partial \boldsymbol{\kappa}_{p}} * \dot{\boldsymbol{\kappa}}_{p}\right) \\
+\frac{\partial g}{\partial \boldsymbol{K}_{p}} *\left(\frac{\partial \boldsymbol{K}_{p}}{\partial \boldsymbol{\Omega}} * \dot{\boldsymbol{\Omega}}+\frac{\partial \boldsymbol{K}_{p}}{\partial \boldsymbol{\kappa}_{t}}+\frac{\partial g}{\partial \boldsymbol{\kappa}_{t}}+\frac{\partial \boldsymbol{K}_{p}}{\partial \boldsymbol{\kappa}_{p}} * \dot{\boldsymbol{\kappa}}_{p}\right) \\
\frac{\partial g}{\partial \boldsymbol{\kappa}_{p}} * \dot{\boldsymbol{\kappa}}_{p}=0
\end{gathered}
$$

which hold only when the plastic deformation continues and the transformation progresses, respectively. Substituting constitutive equations of $\kappa_{i}$ and $\kappa_{p}$, into Eq.(20), one arrives, after straightforward calculation, at the explicit expression of $\dot{\lambda}$ and $\dot{\mu}$ :

where

$$
\dot{\lambda}=L * \dot{\Omega}, \quad \mu=M * \dot{\Omega},
$$

$$
\begin{aligned}
& L=\Delta\left[\left(F_{g} \frac{\partial g}{\partial K_{t}}-G_{g} \frac{\partial f}{\partial K_{t}}\right) * \frac{\partial K_{t}}{\partial \Omega}\right. \\
& \left.+\left(F_{g} \frac{\partial g}{\partial K_{p}}-G_{g} \frac{\partial f}{\partial K_{p}}\right) * \frac{\partial K_{p}}{\partial \Omega}+\left(F_{g} \frac{\partial g}{\partial \Omega}-G_{g} \frac{\partial f}{\partial \Omega}\right)\right] \\
& M=\Delta\left[\left(G_{f} \frac{\partial f}{\partial K_{t}}-F_{f} \frac{\partial g}{\partial K_{t}}\right) * \frac{\partial K_{t}}{\partial \Omega}\right. \\
& \left.+\left(G_{f} \frac{\partial f}{\partial K_{p}}-F_{f} \frac{\partial g}{\partial K_{p}}\right) * \frac{\partial K_{p}}{\partial \Omega}+\left(G_{f} \frac{\partial f}{\partial \Omega}-F_{g} \frac{\partial g}{\partial \Omega}\right)\right], \\
& F_{f}=f_{\boldsymbol{K}_{t}} * \frac{\partial f}{\partial \boldsymbol{K}_{t}}+f_{\boldsymbol{K}_{p}} * \frac{\partial f}{\partial \boldsymbol{K}_{p}}, \quad F_{g}=f_{\boldsymbol{K}_{t}} * \frac{\partial g}{\partial \boldsymbol{K}_{t}}+f_{\boldsymbol{K}_{p}} * \frac{\partial g}{\partial \boldsymbol{K}_{p}},
\end{aligned}
$$




$$
\begin{aligned}
& G_{f}=g_{\boldsymbol{\kappa}_{t}} * \frac{\partial f}{\partial \boldsymbol{K}_{t}}+\boldsymbol{g}_{\boldsymbol{\kappa}_{p}} * \frac{\partial f}{\partial \boldsymbol{K}_{p}}, \quad G_{g}=\boldsymbol{g}_{\boldsymbol{K}_{t}} * \frac{\partial g}{\partial \boldsymbol{K}_{t}}+\boldsymbol{g}_{\boldsymbol{\kappa}_{p}} * \frac{\partial g}{\partial \boldsymbol{K}_{p}}, \\
& \boldsymbol{f}_{\boldsymbol{K}_{t}}=\frac{\partial f}{\partial \boldsymbol{K}_{t}} * \frac{\partial \boldsymbol{K}_{t}}{\partial \boldsymbol{K}_{t}}+\frac{\partial f}{\partial K_{p}} * \frac{\partial \boldsymbol{K}_{p}}{\partial \boldsymbol{K}_{t}}+\frac{\partial f}{\partial \boldsymbol{K}_{t}}, f_{\boldsymbol{K}_{p}}=\frac{\partial f}{\partial \boldsymbol{K}_{t}} * \frac{\partial K_{t}}{\partial \boldsymbol{K}_{p}}+\frac{\partial f}{\partial \boldsymbol{K}_{p}} * \frac{\partial \boldsymbol{K}_{p}}{\partial \boldsymbol{K}_{p}}+\frac{\partial f}{\partial \kappa_{p}}, \\
& \boldsymbol{g}_{\boldsymbol{t}}=\frac{\partial f}{\partial \boldsymbol{K}_{t}} * \frac{\partial \boldsymbol{K}_{t}}{\partial \boldsymbol{\kappa}_{t}}+\frac{\partial f}{\partial \boldsymbol{K}_{p}} * \frac{\partial \boldsymbol{K}_{p}}{\partial \boldsymbol{\kappa}_{t}}+\frac{\partial g}{\partial \boldsymbol{\kappa}_{t}}, \quad \boldsymbol{g}_{\boldsymbol{\kappa}_{p}}=\frac{\partial f}{\partial \boldsymbol{K}_{t}} * \frac{\partial \boldsymbol{K}_{t}}{\partial \boldsymbol{\kappa}_{p}}+\frac{\partial f}{\partial \boldsymbol{K}_{p}} * \frac{\partial \boldsymbol{K}_{p}}{\partial \boldsymbol{\kappa}_{p}}+\frac{\partial g}{\partial \boldsymbol{\kappa}_{p}} \\
& \Delta^{-1}=F_{f} G_{g}-F_{g} G_{f} .
\end{aligned}
$$

The constitutive equations of the internal variables $\kappa_{t}$ and $\kappa_{p}$ are now finally given by

$$
\dot{\kappa}_{t}=\left(\frac{\partial f}{\partial K_{t}} \otimes L+\frac{\partial g}{\partial K_{t}} \otimes M\right) * \dot{\Omega}, \quad \dot{\boldsymbol{\kappa}}_{p}=\left(\frac{\partial f}{\partial K_{p}} \otimes L+\frac{\partial g}{\partial K_{p}} \otimes M\right) * \dot{\Omega}
$$

The thermomechanical and calorimetric constitutive equations are obtained from Eqs.(1), (2) and (22). The result reads

$$
\begin{aligned}
& \dot{\boldsymbol{E}}=\left[\widetilde{E}_{M}+\left(\frac{\partial f}{\partial K_{2}}+\frac{\partial f}{\partial K_{5}}\right) \otimes L+\left(\frac{\partial g}{\partial K_{2}}+\frac{\partial g}{\partial K_{5}}\right) \otimes M\right] * \dot{\boldsymbol{B}}, \\
& \dot{\eta}=\left[\widetilde{E}_{T}+\left(\frac{\partial f}{\partial K_{3}}+\frac{\partial f}{\partial K_{6}}\right) \otimes L+\left(\frac{\partial g}{\partial K_{3}}+\frac{\partial g}{\partial K_{6}}\right) \otimes M\right] * \dot{\boldsymbol{\Omega}},
\end{aligned}
$$

where the ordered sets of the clastic material parameters defined by

$$
\bar{\varepsilon}_{M}=(\boldsymbol{D}, \boldsymbol{\theta}), \quad \bar{\varepsilon}_{T}=\left(\boldsymbol{\theta} / \rho_{0}, c\right)
$$

are used.

The energy balance is rewritten by

$$
\rho_{O} T \dot{\eta}-\left(K_{t} * \dot{\boldsymbol{K}}_{t}+K_{p} * \dot{\kappa}_{p}\right)+\operatorname{Div} \boldsymbol{Q}-\rho_{0} \sigma=0
$$

with the heat production term $\sigma$. If Eqs.(22), (23) and the constitutive equation for the heat flux $Q$ which is not discussed here arc taken into account, Eq.(24) reduces to the heat conduction equation.

Taking into account the constitutive equations (15), the dissipation inequality (6) reduces to

$$
\begin{aligned}
D=\dot{\lambda}\left(\boldsymbol{K}_{t}\right. & \left.* \frac{\partial f}{\partial \boldsymbol{K}_{t}}+\boldsymbol{K}_{p} * \frac{\partial f}{\partial \boldsymbol{K}_{p}}\right) \\
& +\dot{\mu}\left(\boldsymbol{K}_{t} * \frac{\partial g}{\partial \boldsymbol{K}_{t}}+\boldsymbol{K}_{p} * \frac{\partial g}{\partial \boldsymbol{K}_{p}}\right)-\frac{1}{T} \boldsymbol{Q} \cdot \operatorname{Grad} T \geq 0 .
\end{aligned}
$$

Equation(25), together with the convexity condition of the dissipation potential, which should be read in the present context as

$$
K_{t} * \frac{\partial f}{\partial K_{t}}+K_{p} * \frac{\partial f}{\partial K_{p}} \geq 0, \quad K_{t} * \frac{\partial g}{\partial K_{t}}+K_{p} * \frac{\partial g}{\partial K_{p}} \geq 0
$$

claims that in the isothermal case, Grad $T=\mathbf{0}$, the plastic deformation and the transformation processes undergo only when

$$
\dot{\lambda} \geq 0, \quad \dot{\mu} \geq 0
$$

are satisfied, respectively.

\section{TRANSFORMATION KINETICS}

The transformation kinetics is considered in the case of no plastic deformation, which corresponds to the conditions the shape memory alloys are used. Suppose that the transformation condition (13) is simply given by

$$
g=K_{1}+\tilde{g}\left(K_{2}, K_{3}\right)-k(\xi)=0
$$


Equation(16) is now reduced to

$$
\begin{aligned}
& \dot{\xi}=\dot{\mu}=M_{\Sigma}: \dot{\Sigma}+M_{T} \dot{T}, \quad \dot{E}^{*}=\left(\Gamma_{E} \otimes M_{\Sigma}\right): \dot{\Sigma}+\left(\Gamma_{E} M_{T}\right) \dot{T} \\
& \dot{\eta}^{*}=\left(\Gamma_{\eta} M_{\Sigma}\right): \dot{\Sigma}+\left(\Gamma_{\eta} M_{T}\right) \dot{T}, \\
& M_{\Sigma}=\Delta\left(\frac{\partial K_{1}}{\partial \Sigma}+\Gamma_{E}: \frac{\partial K_{2}}{\partial \Sigma}+\Gamma_{\eta} \frac{\partial K_{3}}{\partial \Sigma}\right), M_{T}=\Delta\left(\frac{\partial K_{1}}{\partial T}+\Gamma_{E}: \frac{\partial K_{2}}{\partial T}+\Gamma_{\eta} \frac{\partial K_{3}}{\partial T}\right), \\
& \Delta^{-1}=-\left[\frac{\partial K_{1}}{\partial \xi}+\Gamma_{E}: \frac{\partial K_{2}}{\partial \xi}+\Gamma_{\eta} \frac{\partial K_{3}}{\partial \xi}-\frac{\mathrm{d} k}{\mathrm{~d} \xi}+\left(\frac{\partial K_{1}}{\partial E^{*}}+\Gamma_{E}: \frac{\partial K_{2}}{\partial E^{*}}+\Gamma_{\eta} \frac{\partial K_{3}}{\partial E^{*}}\right): \Gamma_{E}\right. \\
& \left.\quad+\left(\frac{\partial K_{1}}{\partial \eta^{*}}+\Gamma_{E}: \frac{\partial K_{2}}{\partial \eta^{*}}+\Gamma_{\eta} \frac{\partial K_{3}}{\partial \eta^{*}}\right) \Gamma_{\eta}\right]
\end{aligned}
$$

where the coefficients $\Gamma_{E}$ and $\Gamma_{\eta}$ are expressed by

$$
\Gamma_{E}=\frac{\partial \tilde{g}}{\partial K_{2}}, \quad \Gamma_{\eta}=\frac{\partial \tilde{g}}{\partial K_{3}} .
$$

Equation(29) 1 governs the progress of transformation, whereas Eqs.(29)2,3 describe the change in transformation strain and cntropy due to transformation.

Since $\frac{\partial g}{\partial K_{1}}=1$, one gets

$$
\begin{aligned}
& \dot{\xi}=\mu=M_{\Sigma}: \dot{\Sigma}+M_{T} \dot{T} \geq 0 \text { when } \tilde{K}=K_{1}+K_{2}: \Gamma_{E}+K_{3} \Gamma_{\eta} \geq 0, \\
& \dot{\xi}=\mu=M_{\Sigma}: \dot{\Sigma}+M_{T} \dot{T} \leq 0 \text { when } \tilde{K} \leq 0,
\end{aligned}
$$

which leads to the following statement with respect to the classification of the processes: The martensitic transformation progresses if the thermomechanical load $(\Sigma, T)$ moves so that the generalized transformation driving force $\widetilde{K}$ is positive, and the reverse transformation progresses when the generalized driving force is negative.

Just when the transformation starts, the condition (13) reads as

$$
\begin{aligned}
& \left.\left(K_{1}\right)\right|_{j=j_{S}}=\tilde{g}_{S}, \quad \tilde{g}_{s}=k(0)-\left.\tilde{g}\left(K_{2}, K_{3}\right)\right|_{j=j_{S}}, \\
& j_{S}=\left(E_{S}^{e}, T ; 0, \mathbf{0}, 0\right),
\end{aligned}
$$

which can be rephrased in such a way that the transformation starts under a thermomechanical load when the driving force $K_{1}$ reaches a threshold value $\tilde{g}_{S}$. The transformation start condition (32) is represented by a plane in the $\Sigma-T$ space [9.27], which is often observed as a straight line under the uniaxial loading in the shape memory alloys and TRIP steels $[28,29]$.

The same discussion can be performed with regard to the transformation finish condition given by

$$
\begin{aligned}
& \left.\left(K_{l}\right)\right|_{j=j_{f}}=\tilde{g_{f}}, \quad \tilde{g_{f}}=k\left(\xi_{f}\right)-\left.\tilde{g}\left(K_{2}, K_{3}\right)\right|_{j=j_{f}}, \\
& j_{f}=\left(E_{f}^{e}, T ; \xi_{f}, E_{f}^{*}, \quad \eta_{f}^{*}\right),
\end{aligned}
$$

where $\xi_{f}=0.99$ is often chosen in metallurgy as the value of volume fraction at the completion of transformation [30] and $E_{f}^{e}, E^{*} f$ and $\eta_{f}^{*}$ stand for the elastic strain, the transformation strain and the transformation entropy density at that moment, respectively.

Let us employ the Gibbs free energy [31]

$$
\rho_{0} \Psi=-\frac{\Sigma^{2}}{2 E}+3 E \beta_{2} \frac{1-\xi}{\xi} \frac{E^{* 2}}{2}+\rho_{0} \Phi \text { chem,o }-\rho_{0} \xi \Delta \Phi \text { chem },
$$


where $E$ denotes the Young's modulus and $\beta_{2}$ a material parameter relating only to Poisson's ratio. The function $\Phi$ chem,o represents the specific chemical energy in the parent phase while $\Delta \Phi$ chem the difference in the specific chemical encrgy of both phases depending only on the temperature $T$. And the function $\tilde{g}\left(K_{2}, K_{3}\right)$ in Eq.(28) is assumed to have an explicit form

$$
\tilde{g}\left(K_{2}, K_{3}\right)=E_{f}^{*}: K_{2}+\eta^{*} K_{3},
$$

which reduces $\Gamma_{E}=E^{*} f$ and $\Gamma_{\eta}=\eta^{*} f$.

The function $k(\xi)$ in Eq. (28) determines the type of the kinetics in transformation. If its explicit form is given by

$$
k(\xi)=-\frac{1}{k_{0}^{M}} \ln (1-\xi)+k_{00}^{M}
$$

with the constant matcrial parameters $k_{0}^{M}$ and $k_{00}^{M}$, kinctic equation $(29)_{1}$ is reduced to

$$
\dot{\xi}=\left[\frac{1}{k_{o}^{M}(1-\xi)}-3 E \beta_{2}\left(E^{*} f\right)^{2}\right]^{-1}\left[E_{f}^{*}: \dot{\Sigma}+\rho_{O}\left(\eta_{f}^{*}-\Delta S \text { chem }\right) \dot{T}\right],
$$

where $\Delta S$ chem $=-\frac{\mathrm{d} \Delta \Phi \text { chem }}{\mathrm{d} T}$ denotes a difference in the specific entropy of both phases. The term $3 E \beta_{2}\left(E^{*} f\right)^{2}$ stems from the interaction energy term between the phases in the Gibbs frec energy, the second term in the right-hand side in Eq.(34).

For the case of no interaction energy term in the Gibbs free energy, Eq.(37) reduces to

$$
\frac{\dot{\xi}}{1-\xi}=k_{o}^{M}\left[E_{f}^{*}: \dot{\Sigma}+\rho_{0}\left(\eta_{f}^{*}-\Delta S_{\mathrm{chem}}\right) \dot{T}\right],
$$

which becomes simpler, if one takes into account the fact that the martensitic transformation starts at the martensite start temperature $M_{S}$ under the stress-free state, to give a solution

$$
\xi=1-\operatorname{cxp}\left[-k_{0}^{M} E_{f}^{*}: \Sigma-k_{0}^{M} \rho_{0}\left(\eta^{*} f-\Delta S \text { chem }\right)\left(T-M_{S}\right)\right] .
$$

Equation(39) is an extended 3-D version of the Koistinen-Marburger [32] and Magee [34] kinetics for the stress-free case.

The transformation start plane (32) is now given by

$$
E^{*} f: \Sigma+\rho_{O}\left(\eta^{*} f-\Delta S \text { chem }\right)\left(T-M_{S}\right)=0 .
$$

In the case of the reverse transformation, the form of the function $k(\xi)$ may be selected as

$$
k(\xi)=-\frac{1}{k_{0}^{A}} \ln \xi+k_{00}^{A}
$$

to give a transformation kinctics of the form

$$
\xi=\operatorname{cxp}\left[-k_{O}^{A} E_{f}^{*}: \Sigma-k_{o}^{A} \rho_{O}\left(\eta^{*} f-\Delta S \mathrm{chem}\right)\left(T-A_{S}\right)\right] \text {. }
$$

\section{References}

Müllcr,I. and Xu,H.B., Acta metall. et material, 39 (1991) 263-271.

2] Huo, Y. and Müller,I., Continuum Mech. Thermodyn., 5 (1993) 163-204.

3] Raniccki,B., Lexcellent,Ch. and Tanaka,K., Arch. Mech., 44 (1992) 261-284.

$4]$ Levitas, V.I., Mcch. Rescarch Commun., 21 (1994) 273-280.

5] Heidug,W., and Lehner,F.K., Pure Appl. Geophysics, 13 (1985) 91-98.

[6] Abeyaratne,R. and Knowles, J.K., J. Mech. Phys. Solids, 38 (1990) 345-360.

[7] Abcyaratnc,R. and Knowles,J.K., J. Mech. Phys. Solids, 41 (1993) 541-571.

Raniccki,B. and Tanaka,K., Int. J. Engng Sci., 32 (1994) 1845-1858. 
(9] Tanaka,K., Kobayashi,S. and Sato,Y., Int. J. Plasticity, 2 (1986) 59-72.

[10] Brandon,D. and Rogers, R.C., J. Intell. Material Syst. Structures, 3 (1992) 255-267.

[11] Brinson,L.C., J. Intell. Material Syst. Structures, 4 (1993) 229-242.

12] Fischer,F.D. and Tanaka,K., Int. J. Solids Structures, 29 (1992) 1723-1728.

13] Sun,Q.P., Hwang,K.C. and Yu,S.W., J. Mech. Phys. Solids, 39 (1991) 507-524.

[14] Sun,Q.P. and Hwang, K.C., J. Mech. Phys. Solids, 41 (1993) 1-17.

[15] Tanaka,K., Oberaigner,E.R.. and Fischer,F.D., A unified theory on thermomechanical mesoscopic behavior of alloy materials in the process of martensitic transformation, L.C.Brinson and B.Moran, Eds., Mechanics of Phase Transformations and Shape Memory Alloys (ASME, New York, 1994) $151-157$.

[16] Tanaka,K. and Nagaki,S., Ing.-Arch., 51 (1982) 287-299.

[17] Wayman,C.M., Phase transformations, nondiffusive, R.W.Cahn and P.Haasen, Eds., Physical Metallurgy, third, revised and enlarged ed. (North-Holland Physics Publishing, Amsterdam, 1983) Chap.15.

[18] Kaufman,L. and Hillert,M., Thermodynamics of martensitic transformations, G.B.Olson and W.S.Owen, Eds., Martensite, (ASM International, 1992) Chap.4.

[19] Lemaitre,J. and Chaboche,J.-L., Mechanics of Solid Materials (Cambridge University Press, Cambridge, 1990).

[20] Edclen,D.G.B., Int. J. Engng Sci., 12 (1974) 121-141.

[21] Eringen,A.C., Thermodynamics of Continua, A.C.Eringen, Ed., Continuous Physics (Academic Press, New York, 1975) Part I-3.

[22] Peric,D., Int. J. Num. Methods Engng, 36 (1993) 1365-1393.

[23] Raghavan,V., Kinetics of martensitic transformations, G.B.Olson and W.S.Owen, Eds., Martensite, (ASM International, 1992) Chap.11.

[24] Wang,Z.G. and Inoue,T., J. Soc. Materials Sci., Japan, 32, 991-996.

[25] Leblond,J.B., Devaux,J. and Devaux,J.C., Int. J. Plasticity, 5, 1989, 551-572.

[26] Funakubo,H., Ed., Shape Memory Alloys (Gordon and Breach Science Publishers, New York, 1987).

[27] Tanaka,K., Analysis of recovery stress and cyclic deformation in shape memory alloys, O.Brüller et al., Eds., Advances in Continuum Mechanics (Springer-Verlag, Berlin-Heidelberg-New York, 1991) 441-451.

[28] Tamura,I., Metal Sciences, 16 (1982) 245-253.

[29] Wayman,C.W., and Duerig,T.W., An introduction to martensite and shape memory, T.W.Ducrig et al., Eds., Enginecring Aspects of Shape Memory Alloys (Butterworth-Heinemann, London, 1990) 3-20.

[30] Porter,D.A. and Easterling,K.E., Phase Transformations in Metals and Alloys, (Van Nostrand Reinhold (International) Co. Ltd, Wokingham, 1981).

[31] Fischer,F.D., Berveiller,M., Tanaka,K. and Oberaigner,E.R., Arch. Appl. Mech., 63 (1994) 54-85.

[32] Koistinen,D.P. and Marburger,R.E., Acta Metall., 7, 59.

[33] Magec,C.L., The nucleation of martensite, H.I.Aaronson, Ed., Phase Transformations (AMS, Metals Park, 1969) 115-156. 